\title{
PESQUISAS DE USOS DO TEMPO: UM INSTRUMENTO PARA AFERIR AS DESIGUALDADES DE GÊNERO
}

\author{
DANIELA PEIXOTO RAMOS
}

Universidade de Brasília

\begin{abstract}
Resumo: Busca-se, neste artigo, analisar a interface entre desigualdades de gênero e usos do tempo, situando esta análise na perspectiva do desenvolvimento das pesquisas de usos do tempo e observando como se materializa nos indicadores que compóem o Retrato das desigualdades de gênero e raça. Inicialmente, são caracterizadas as pesquisas de usos do tempo, recuperando-se um pouco de seu histórico e estágio atual. Discute-se, também, a perspectiva teórica que enfatiza o gênero como categoria central para análise dos usos do tempo. Apresentam-se alguns dos principais resultados encontrados por pesquisas de usos do tempo em nível internacional e enfatizam-se os indicadores de usos do tempo produzidos a partir da Pesquisa Nacional por Amostra de Domicílios. Finalmente, sugere-se que as pesquisas de usos do tempo podem contribuir para o fomento de políticas públicas destinadas a combater as desigualdades de gênero.
\end{abstract}

Palavras-chove: uso do tempo; gênero; desigualdade; trabalho não remunerado.

\section{Introdução}

A análise dos chamados "usos do tempo", isto é, de como as pessoas empregam seu tempo, distribuindo esse recurso escasso e universal entre as mais diversas atividades, pode elucidar importantes formas de manifestação das desigualdades de gênero e raça. Os padrões de usos do tempo apresentados pelos segmentos sociais são moldados por estruturas sociais, normas culturais e arranjos institucionais específicos a cada sociedade. É assim que o Retrato das desigualdades de gênero e raça, ${ }^{1}$ cujos dados são objeto do dossiê de que faz parte este artigo, tem como um de seus blocos temáticos as dimensões de usos do tempo captadas pela Pesquisa Nacional por Amostra de Domicílios (PNAD), do

Copyright (C) 2009 by Revista Estudos Feministas.

' Luana PINHEIRO et al., 2008. 
Instituto Brasileiro de Geografia e Estatística (IBGE). Busca-se, neste artigo, analisar a interface entre desigualdades de gênero e usos do tempo, situando esta análise na perspectiva do desenvolvimento das pesquisas de usos do tempo e observando como se materializa nos indicadores que compõem o Retrato.

Na primeira seção, descrevem-se os parâmetros das pesquisas de usos do tempo, caracterizando o estágio atual da produção de tais pesquisas. Em seguida, apresenta-se a perspectiva teórica que enfatiza o gênero como categoria central para análise dos usos do tempo, em especial do tempo destinado ao trabalho não remunerado. Na seção seguinte, discutem-se alguns dos principais resultados encontrados por pesquisas de usos do tempo e analisam-se, sucintamente, alguns dados referentes ao Brasil. Ao final, tecem-se algumas considerações conclusivas.

\section{Breve caracterização e histórico das pesquisas de usos do tempo}

Pesquisas de usos do tempo almejam captar comportamentos não perceptíveis por pesquisas domiciliares tradicionais, as quais não adentram o universo intradomiciliar e, portanto, não desvelam como ocorre a repartição de trabalho e de recursos, entre eles o tempo, na esfera privada - uma preocupação central dos estudos de gênero. O que tais pesquisas fazem é captar empiricamente, utilizando diferentes métodos, as atividades em que as pessoas empregam seu tempo durante um período específico, em geral, um dia. $O$ objetivo é, assim, traçar um perfil dos chamados "orçamentos de tempo" individuais, que compreendem a "sequência, o horário e a duração das atividades realizadas pelos indivíduos". ${ }^{2}$ Adicionalmente, são caracterizados também outros aspectos contextuais, tais como uma descrição de cada episódio por parte do respondente, onde ocorreu, com quem a atividade foi executada ou que outras pessoas estavam presentes e, em alguns casos, "em benefício de quem" as atividades foram realizadas.

É justamente essa inédita riqueza de dados empíricos sobre a vida cotidiana, levantada por pesquisas de usos do tempo, que tem levado alguns autores a caracterizálas como "revolucionárias" 3 para o estudo do comportamento e da mudança social, de utilidade para qualquer área do comportamento humano. Confirmando esta última percepção, há, atualmente, uma profusão de estudos que utilizam dados de usos do tempo nas mais diversas áreas.

Ao buscar os antecedentes das pesquisas de uso do tempo tal como caracterizadas acima, encontra-se como primeiro exemplo citado pela literatura um pequeno estudo feito na Universidade de Colúmbia sobre como trabalhadores usam seu tempo livre. ${ }^{4} \mathrm{O}$ primeiro grande estudo multinacional de uso do tempo foi realizado entre 1965 e 1966, dirigido pelo sociólogo húngaro Alexander Szalai e implementado em 12 países, com 30 mil entrevistados no total. A publicação resultante - O uso do tempo - é considerada uma referência obrigatória na área, tendo criado padrões metodológicos referenciais.

Europa Ocidental, Estados Unidos, Canadá e Austrália se notabilizam pela produtividade dessa área de estudos. Na América Latina, dez países já possuem pesquisas de usos do tempo aplicadas por seus institutos de estatística nacionais: Cuba, México, Equador, Uruguai, Chile, Nicarágua, Guatemala, Costa Rica, Bolívia e República Dominicana, e agora também será a vez do Brasil. Destacam-se, aqui, alguns estudos

\footnotetext{
${ }^{2}$ Elke AMMASSARI, 2000, p. 3154.

${ }^{3}$ William MICHELSON, 2005, p. 17.

${ }^{4}$ George BEVANS, 1913.
} 
subnacionais já realizados. ${ }^{5}$ Essa proliferação de pesquisas é resultado, em parte, da prioridade de investigação dada pela ONU à "medição do tempo e sua utilização", como resultado das conferências ocorridas durante a Década da Mulher: 1975-1985.

\section{Aspectos metodológicos}

As pesquisas de usos do tempo podem ter desenhos diferentes visto estarem focadas num segmento populacional específico ou num grupo de atividade em particular, como 0 trabalho não remunerado, e abrangerem períodos de tempo diversos - apenas um dia considerado típico, ou um dia da semana e um domingo, ou diferentes períodos ao longo do ano - para captar sazonalidades.

Em relação ao método de coleta dos dados, estabeleceu-se como boa prática a utilização de um diário, dividido em unidades de tempo padronizadas e pequenas, como cinco ou dez minutos, associadas a atividades descritas pelo respondente em seus próprios termos e, em seguida, codificadas. No diário, o entrevistado é solicitado a reportar suas atividades "primárias", isto é, as atividades consideradas principais, em cada período de tempo, e também as "secundárias", realizadas concomitantemente, como, por exemplo, cozinhar e, ao mesmo tempo, cuidar de crianças. Em geral, a análise considera apenas as atividades primárias, o que tende a ser criticada porque se deixa de considerar as estratégias de maximização do tempo ou se deixa de considerar atividades secundárias relevantes de cuidado, como supervisão de pessoas dependentes.

O uso do diário como método de coleta de dados é, em geral, considerado mais adequado do que a realização de questões diretas sobre a quantidade de tempo alocada para atividades diversas. Isso porque o diário possibilita estimativas de tempo mais precisas, minimiza o viés de respostas orientadas por percepções sobre comportamento socialmente desejado e permite captar melhor atividades fragmentadas e outros aspectos, como o ritmo, a duração das atividades em separado, as variações entre dias ou períodos, além de outras possibilidades analíticas que só o diário proporciona. Uma das desvantagens é que ele torna a pesquisa mais complexa e trabalhosa.

O problema da falta de uniformidade na classificação das atividades e também da falta de comparabilidade das pesquisas foi minorado com a proposta da Classificação Internacional de Atividades para Estatísticas de Usos do Tempo (International Classification of Activities for Time-Use Statistics - ICATUS) pela Divisão de Estatística das Nações Unidas em 1997. A quantidade de categorias utilizada atualmente é muito maior do que quando foram produzidas as primeiras pesquisas, em razão da necessidade de dar conta da crescente heterogeneidade de estilos de vida em diferentes culturas. Ainda assim, resta a crítica de que a codificação é feita pelo pesquisador, e não pelo entrevistado, o que pode levar à divergência de sentidos atribuídos à atividade.

O método a ser implementado pelo IBGE envolve duas entrevistas: uma para captação de dados sociodemográficos e instrução do entrevistado sobre o preenchimento do diário; e uma segunda visita, no dia seguinte ao dia de referência sorteado, em que o entrevistador questiona o respondente sobre o diário e registra as respostas tal como aparecem num coletor eletrônico.

\footnotetext{
${ }^{5}$ Múltiplas temporalidades de referência: análise dos usos do tempo entre grupos domésticos na população de Belo Horizonte. Esse estudo foi realizado em 2001 e coordenado pela Prof. ${ }^{a}$ Neuma Aguiar. Há também o estudo realizado pelo Centro Brasileiro de Análise e Planejamento (CEBRAP), em 2005, intitulado O uso do tempo livre e as práticas culturais na RMSP e coordenado por Isaura Botelho.
} 


\section{Analisando os usos do tempo com foco nas relações de gênero e na divisão sexual de trabalho}

Ao contrário de outras perspectivas teóricas relativas à distribuição do trabalho doméstico, ${ }^{6}$ a perspectiva de gênero supõe que a distribuição de trabalho não remunerado intradomiciliar é majoritariamente explicada pela forma como se estabelecem as relações de gênero a partir da divisão sexual de trabalho, a qual reserva ao homem o trabalho remunerado para o mercado (e também a participação na esfera pública de forma geral) e à mulher, as atividades domésticas limitadas à esfera privada. Não é possível entender a distribuição de trabalho não remunerado em âmbito intradomiciliar nem tampouco a divisão entre ocupações femininas e masculinas no mercado de trabalho sem recorrer à literatura que trata de como se estruturam as relações sociais de gênero. O trabalho não remunerado e sua associação com papéis de mãe e esposa é um grande estruturador da própria identidade feminina, especialmente para mulheres de gerações mais antigas, que tiveram uma socialização tradicional e não chegaram a assumir outros papéis sociais mais modernos. No entanto, observa-se que mesmo as mulheres jovens, que vivem com maior intensidade um processo de "reestruturação das relações de gênero nas mais variadas dimensões da vida social"7 que aponta para a difusão de valores mais igualitários nas relações de gênero, ainda têm um longo caminho a percorrer nesse sentido.

É com base no argumento da persistência da divisão sexual de trabalho em âmbito doméstico que são explicados alguns dos principais resultados dos estudos de usos do tempo, tais como o fato de que as mulheres, quando vivem com companheiros, exercem muito mais trabalho não remunerado do que quando vivem sozinhas, mesmo na ausência de filhos. Isso explicaria também por que os homens não aumentam substancialmente sua carga de trabalho não remunerado mesmo quando suas esposas exercem trabalho remunerado, tal como mostra o Retrato das desigualdades. ${ }^{8}$ Segundo essa perspectiva, portanto, o principal determinante da repartição doméstica de tempo dedicado ao trabalho é a categoria "gênero", que, no âmbito desta análise, se entende como uma "construção social, cultural e psicológica que se impõe sobre as diferenças biológicas" "relacionadas ao sexo. Embora essa construção varie entre culturas, ela sempre faz referência à diferença sexual (distinção entre homem e mulher, macho e fêmea), segundo Adriana Piscitelli, ${ }^{10}$ e é indispensável considerá-la porque permite compreender as relações sociais.

Para corroborar com essa perspectiva, a pesquisa seminal coordenada por Alexander Szalai, " que comparou países tão distintos quanto Estados Unidos e União Soviética numa época em que os modos de produção e o regime político eram muito diferentes, encontrou padrões bem divergentes de usos do tempo, porém com os mesmos tipos de desigualdades de gênero, embora com intensidades variadas. No entanto, é preciso agregar outras variáveis para explicar as diferenças entre os padrões de usos do tempo encontrados em diversos países. Elas podem ser devidas, por exemplo, às particularidades dos processos de desenvolvimento de cada sociedade, que têm claros impactos sobre como os diferentes estratos sociais usam seu tempo.

\footnotetext{
${ }^{6}$ Por exemplo, as perspectivas da disponibilidade de tempo e dos recursos relativos, citadas por Zuleika FERRE, Giorgina PIANI e Máximo ROSSI, 2004.

${ }^{7}$ Bila SORJ, 2005, p. 80.

${ }^{8}$ PINHEIRO et al., 2008.

9 Judith SHAPIRO, 1981 apud Adriana PISCITELLI, 1997, p. 50.

10 PISCITELLI, 1997.

${ }^{11}$ Alexander SZALAI, 1972.
} 
Sendo assim, para entender como as desigualdades de gênero em termos sociais, econômicos e políticos produzem diferenças em termos de usos do tempo, é preciso, como nota Elke Ammassari, ${ }^{12}$ levar em conta variáveis relativas à composição familiar, à estrutura do mercado de trabalho, à disponibilidade de oferta de trabalho doméstico assalariado, ao regime de políticas públicas predominante, ao padrão de distribuição de renda e riqueza, entre outras. Portanto, deve-se considerar que os usos do tempo por homens e mulheres são, em grande medida, constrangidos por sua inserção social em termos de gênero, raça e classe, e, como tal, são relativamente estáveis. Ao lado disso, têm-se, também, os arranjos institucionais formais e informais, que constrangem os usos do tempo e induzem à permanência das desigualdades de gênero. Esses arranjos variam entre sociedades e mudam ao longo do tempo, mesmo que de forma lenta e gradual, não se completando no intervalo de apenas uma ou duas gerações. ${ }^{13}$

Até agora, o foco central das investigações de usos do tempo na área de gênero tem sido o trabalho não remunerado devido à relevância desse tema para a agenda feminista, que procura dimensionar o tempo dedicado a ele, em grande parte por mulheres, e traduzi-lo em termos econômicos para evidenciar sua magnitude ${ }^{14} \mathrm{e}$, assim, tentar evitar que continue sendo subordinado ao tempo de trabalho remunerado. Ao lançar luz sobre o trabalho não remunerado, as análises de usos do tempo têm pretendido, também, explicitar o quão importantes são as executoras desse trabalho para a provisão de bem-estar social e o quão deletérias são as consequências da dedicação ao trabalho não remunerado, especialmente em termos de perda de autonomia, escassez de tempo, pobreza e falta de acesso a espaços sociais e a direitos em geral, como demonstra Susan Okin. ${ }^{15}$

Daí a necessidade de conceituar e distinguir os diferentes tipos de trabalho não remunerado, como propõe Rosario Aguirre: ${ }^{16}$ o trabalho doméstico, o trabalho de cuidados familiares, o trabalho voluntário ou a serviço da comunidade e o de subsistência, cada qual com suas particularidades. A compreensão das características específicas dos subtipos de trabalho não remunerado contribui para o aperfeiçoamento dos instrumentos de captação do tempo dedicado a cada subtipo e para a elaboração de análises mais ricas a partir delas, que relacionem, por exemplo, os usos do tempo e as percepções sobre eles com o exercício da cidadania social e política, com a mudança dos papéis de gênero e com as relações de poder intradomiciliares.

O tempo destinado ao trabalho remunerado, exercido no âmbito do mercado, tende a ser o mais institucionalizado, isto é, regulado por normas e convenções que regem sua duração. Por outro lado, o tempo dedicado ao trabalho não remunerado, apesar de rígido, ou seja, determinado pelos horários das instituições com as quais interage (escolares, de saúde, de transporte etc.), está menos sujeito a intervenções regulatórias externas. Como os

\footnotetext{
12 AMMASSARI, 2000.

${ }^{13}$ No que diz respeito ao reflexo da permanência das desigualdades de gênero em termos de usos do tempo, há uma importante proposição feita por Jonathan Gershuny (1995) segundo a qual estaria havendo um processo de "adaptação lenta e defasada" (lagged adaptation) em direção à equidade de gênero em termos da divisão sexual de trabalho. Para compreender esse processo, segundo o autor, importa considerar as estratégias individuais ou familiares de mudanças de comportamento e de aquisição de novas habilidades relacionadas aos afazeres domésticos, no caso dos homens.

${ }^{14}$ A Comissão de Estatísticas da ONU revisou o sistema de contas nacionais em 1993, para incorporar as chamadas "contas satélites", que incluem o valor do trabalho não remunerado. Desde então, tem havido esforços em diversos países em quantificar o percentual do PIB representado pelo trabalho não remunerado. As estimativas, utilizando diferentes métodos, vão desde $21,7 \%$ no México em 2002 até 60,7\% na Espanha segundo Soledad SALVADOR (2009).

15 Susan OKIN, 1998.

16 Rosario AGUIRRE, 2009.
} 
trabalhos remunerado e não remunerado estão, na prática, vinculados, o tempo que sobra para a reprodução social, como coloca Cláudio Dedecca, ${ }^{17}$ é definido em função da carga horária que se institui para o tempo econômico, que tem precedência sobre o primeiro, por ser remunerado e associado.

\section{Principais resultados}

Esta seção apresenta, de forma sumária, alguns dos principais resultados de pesquisas de usos do tempo, com enfoque no interesse central deste artigo: sua relação com desigualdades de gênero. Em primeiro lugar, apresentam-se algumas conclusões a que chegaram pesquisas realizadas em sociedades que podem ser denominadas pósindustriais, que também são aquelas que dispõem da maior quantidade de dados e análises de usos do tempo, como já mencionado: Estados Unidos, Canadá, países da Europa Ocidental e Austrália. Em seguida, discutem-se os dados presentes no Retrato das desigualdades, tentando-se estabelecer algumas comparações entre as tendências observadas para os países desenvolvidos e os indicadores para o Brasil.

As pesquisas feitas em países desenvolvidos mostram que os usos do tempo para os trabalhos remunerado e não remunerado estão vinculados, visto que quanto mais tempo as mulheres se dedicam ao trabalho remunerado, menos tempo é alocado por elas ao não remunerado, e que essa tendência tem crescido ao longo dos anos. Além da posição na ocupação, outras variáveis significativas para explicar as diferenças no tempo de trabalho não remunerado são idade, presença de filhos, idade dos filhos, escolaridade da mulher, estado civil e renda domiciliar.

Os resultados de algumas pesquisas longitudinais ${ }^{18}$ revelam que os homens aumentam de forma apenas residual, ainda que contínua, o seu tempo de trabalho doméstico, em resposta ao incremento da participação feminina no mercado de trabalho. No entanto, o resultado mais significativo e aceito é o de que as mulheres têm se mostrado capazes de adaptações mais rápidas visto reduzirem significativamente, em torno de $20 \%$, como demonstram Michael Bittman e George Matheson ${ }^{19} \mathrm{em}$ dados referentes à Alemanha, sua dedicação ao trabalho doméstico quando entram no mercado de trabalho.

Também é interessante notar que, na literatura, revisada por Jonathan Gershuny e Oriel Sullivan, ${ }^{20}$ de usos do tempo referentes aos últimos 30 anos em países desenvolvidos, tanto mulheres quanto homens têm aumentado o tempo destinado ao cuidado com crianças e que as mulheres tiveram um ligeiro aumento do tempo dedicado ao lazer, ao passo que os homens apresentaram um decréscimo. Esses resultados sugerem que estaria havendo uma gradual redução das desigualdades de gênero, em termos da divisão do trabalho não remunerado, ainda que isso se deva mais à redução da carga de trabalho não remunerado pelas mulheres do que ao comportamento dos homens. A despeito disso, ainda é grande a assimetria de usos do tempo entre os sexos.

No Brasil, embora ainda não tenha havido uma pesquisa de abrangência nacional sobre usos do tempo, a PNAD incorpora, desde 1992, algumas questões importantes sobre realização de afazeres domésticos e tempo médio de deslocamentos entre a residência e o local de trabalho, e, a partir da PNAD 2001, tempo médio dedicado a afazeres domésticos.

\footnotetext{
17 Cláudio DEDECCA, 2004.

${ }^{18}$ As pesquisas usadas por Jonathan GERSHUNY, Michael BITTMAN e John BRICE (1997) são German SocioEconomic Panel, British Household Panel Study e US Panel Study of Income Dynamics.

${ }^{19}$ Michael BITTMAN e George MATHESON, 1996.

20 Jonathan GERSHUNY e Oriel SULLIVAN, 2003.
} 
No que se refere ao tempo de deslocamento, a desigualdade marcante é a de raça, visto que as diferenças entre homens e mulheres não são significativas, como demonstrado no Retrato das desigualdades, cujos dados são usados nesta seção. Durante todo o período de 1996 a 2007, os percentuais da população branca, cujo tempo médio de deslocamento é de até 30 minutos, superaram os percentuais da população negra (com uma média de $71 \%$ da população branca e de $66 \%$ da negra), ao passo que, nos tempos de deslocamento maiores, de 30 minutos até 1 hora e de 1 a 2 horas, os negros predominam (média de $21 \%$ de brancos e $24 \%$ de negros e de $6 \%$ de brancos e $8 \%$ de negros, respectivamente).

A discrepância de gênero em termos de usos do tempo para afazeres domésticos permaneceu enorme durante todo o período de 1996 a 2007, considerando a população de 16 anos ou mais de idade: a proporção da população dessa faixa etária que cuida de afazeres domésticos variou de $44,4 \%$ para homens e $92,6 \%$ para mulheres no primeiro ano da série, em que a discrepância foi a maior, até 50,7\% para homens e $89,9 \%$ para mulheres em 2007. Em outros países latino-americanos (Bolívia, em 2001, Guatemala, em 2000, Equador, em 2004, Nicarágua, em 1998, e México, em 2003, segundo Vivian Milosavlejvic e Odette Tacla, ${ }^{21}$ que utilizaram dados de recentes pesquisas de usos do tempo), os resultados são semelhantes: a taxa de mulheres que participam das atividades domésticas é de mais de $90 \%$, ao passo que a dos homens é, na média, $64 \%$.

Por um lado, o fato de que a taxa de participação nas atividades domésticas pelos homens tenha crescido durante o período citado pode indicar uma "adaptação defasada" também no Brasil, para usar os termos de Gershuny ${ }^{22}$ já mencionados. Por outro lado, sempre segundo os indicadores do Retrato, a média de tempo dedicado por homens a afazeres domésticos praticamente não se alterou no período: ela passou de 11,2 em 2001 para 10,6 horas semanais em 2007 para a população masculina em geral, e o mesmo se repete para a população masculina desocupada e inativa (cujas médias de horas declinaram de 14,7 para 13,9 e de 14,7 para 13,7, portanto, uma redução de uma hora entre os anos de 2001 e 2007, respectivamente). Como ainda não temos dados mais detalhados sobre o tempo dedicado a diferentes tipos de trabalho não remunerado, não é possível saber se no Brasil o tempo que os homens dedicam ao cuidado com crianças teria também aumentado, como ocorrido em países desenvolvidos, tal como mencionado.

A média de horas semanais dedicadas a afazeres domésticos pelas mulheres de 16 anos ou mais de idade foi diminuindo continuamente ao longo do período, tendo passado de 30,9 em 2001 para 27,2 horas semanais em 2007. Uma possível explicação para isso poderia ser de que as mulheres brasileiras, assim como as de países desenvolvidos, estariam reduzindo seu tempo de dedicação ao trabalho não remunerado ao aumentar sua participação no mercado de trabalho. ${ }^{23} \mathrm{O}$ indicador para a população feminina ocupada na faixa etária de 16 anos ou mais reforça essa hipótese: eram 24,4 horas semanais dedicadas aos afazeres domésticos em 2001, que decresceram continuamente até chegar a 22,3 horas em 2007, portanto, bem inferiores aos números, citados acima, referentes à população feminina em geral.

Também as mulheres sem ocupação e inativas tiveram seu tempo médio de afazeres domésticos reduzido ao longo do período 2001 a 20007: entre as primeiras, o tempo médio

\footnotetext{
${ }^{21}$ Vivian MILOSAVLEJIC e Odette TACLA, 2008.

22 GERSHUNY, 1995.

${ }^{23} \mathrm{O}$ indicador de taxa de participação no mercado de trabalho da população feminina de 16 anos ou mais de idade, presente no Retrato das desigualdades, revela que, no mesmo período, isto é, de 2001 a 2007, as mulheres tiveram sua taxa aumentada de $55 \%$ para $58,6 \%$.
} 
semanal passou de 33,7 para 29,5 horas e, entre as inativas, que são a categoria que mais se dedica ao trabalho doméstico, as horas semanais declinaram de 37,4 para 32,9. De qualquer forma, ainda é grande a assimetria de gênero entre inativos: as mulheres inativas têm uma dedicação de 19 horas superior à dos homens na mesma condição, ainda que eles realizem mais afazeres domésticos do que os homens ocupados (uma diferença de quase 5 horas a mais para os primeiros em 2001, que se reduziu para 4 horas a mais em 2007). Esse fenômeno de redução das horas semanais dedicadas a afazeres domésticos por mulheres que participam ou não do mercado de trabalho pode se dever a transformações ocorridas na própria forma de execução de afazeres domésticos, com a incorporação de novos aparelhos eletrodomésticos poupadores de tempo, como máquina de lavar e geladeira, ${ }^{24}$ ou a mudanças nas percepções e nos comportamentos de mulheres em relação a trabalho doméstico, ${ }^{25}$ ou ainda, como sugerem Luana Pinheiro et al. ${ }^{26}$ à contratação de trabalho doméstico remunerado.

No Brasil, como visto, o tempo de dedicação das mulheres aos afazeres domésticos correspondeu a mais do que o dobro do tempo masculino durante todo o período de 1996 a 2007. Embora as mulheres estejam se mostrando, também aqui, capazes de mudar seu comportamento de maneira mais rápida do que os homens - já que são elas que reduzem seu tempo de trabalho não remunerado quando também trabalham fora, ao passo que os homens praticamente não mudam -, as desigualdades de gênero, aferidas pelos indicadores de usos do tempo, persistem.

\section{Considerações finais}

As possibilidades que oferecem as pesquisas de usos do tempo são várias. Diversas áreas poderiam se beneficiar do arcabouço teórico-metodológico já construído e das bases de dados disponíveis. No que se refere ao enfrentamento das desigualdades de gênero e raça, as análises podem ajudar a compreender questões relativas ao acesso e à utilização de serviços sociais por parte de grupos que têm constrangimentos temporais distintos. Ao mesmo tempo, elas evidenciam, também, que o desenho e a implementação de políticas públicas devem estar pautados pela compreensão dos impactos desiguais dessas políticas sobre homens e mulheres na esfera familiar, tendo em vista sua divisão interna de trabalho e seu modo de prover bem-estar social a seus membros. Assim, as pesquisas de usos do tempo contribuem para a incorporação de novos temas à agenda de políticas públicas, como a necessidade de maior provisão de serviços sociais de cuidado em virtude da sobrecarga que têm sofrido as mulheres.

Em termos de aferição das desigualdades de gênero, a sucinta análise apresentada neste artigo pretende sugerir que a incorporação dos dados de usos do tempo pode ilustrar aspectos relativos à divisão sexual de trabalho que não são específicos da realidade

\footnotetext{
${ }^{24}$ A proporção de domicílios que não possuem máquina de lavar, segundo dados do Retrato, decresceu de $66,4 \%$ em 2001 para $60,5 \%$ em 2007. No caso dos domicílios chefiados por mulheres, a proporção foi de $67,6 \%$ em 2001 para 59,9\% em 2007. Os dados relativos à geladeira, outro eletrodoméstico poupador de tempo de grande relevância, revelam que esse é um aparelho muito mais comum. A proporção dos domicílios que não a possuem reduziu-se de 15,1\% em 2001 para 9,2\% em 2007, e, para domicílios chefiados por mulheres, a proporção variou de $13,8 \%$ para $7,6 \%$.

${ }^{25}$ A esse respeito, é interessante observar os resultados de um survey nacional de atitudes, opiniões e práticas conduzido em 2003 e coordenado por Clara ARAÚJO e Celi SCALON (2005) sobre vários aspectos das relações de gênero. A título de exemplo, tem-se que $81,6 \%$ das mulheres (e $70,2 \%$ dos homens) concordam com a afirmação de que "os homens deveriam dividir igualmente todas as tarefas domésticas".

${ }^{26}$ PINHEIRO et al., 2008.
} 
brasileira. A despeito da escassez atual de dados nacionais sobre usos do tempo, as possibilidades de análise, para as quais o Retrato das desigualdades de gênero e raça tem o mérito de chamar atenção, foram apenas tangenciadas neste artigo. Em breve, elas crescerão de forma exponencial, já que o IBGE disponibilizará todo um banco de dados para análises mais profundas, tanto intranacionais quanto comparativas em nível internacional.

\section{Referências bibliográficas}

AGUIRRE, Rosario. "Uso del tiempo y desigualdades de género en el trabajo no remunerado". In: AGUIRRE, Rosario (Ed.). Las bases invisibles del bienestar social. Montevideo: Doble clic editoras, 2009. p. 23-86.

AMMASSARI, Elke. "Time Use Research." In: BORGATTA, Edgar; MONTGOMERY, Rhonda (Eds.). Encyclopedia of Sociology. New York: Macmillan Reference, 2000. v. 5. p. 3153-3165.

ARAÚJO, Clara; SCALON, Celi (Orgs.). Gênero, família e trabalho no Brasil. Rio de Janeiro: FGV, 2005.

BEVANS, George. How Workingmen spend their Spare Time. New York: Columbia University Press, 1913.

BITTMAN, Michael; MATHESON, George. "All Else Confusion: What Time Use Surveys show about Changes in Gender Equity." Social Policy Research Centre Discussion Paper, Sidney: University of South Wales, n. 72, p. 1-27, 1996.

DEDECCA, Cláudio. "Tempo, trabalho e gênero". In: COSTA, Ana et al. (Orgs.). Reconfiguração das relações de gênero no trabalho. São Paulo: CUT Brasil, 2004. p. 21-52.

FERRE, Zuleika; PIANI, Giorgina; ROSSI, Máximo. El tiempo en el hogar: parejas desparejas. Montevideo: Universidad de la República, 2004. Documento de trabajo número 18/04.

GERSHUNY, Jonathan. "Change in the Division of Domestic Work: Micro-sociological Evidence." DIW Working Papers, Berlim, Draft. 1, p. 1-20, 1995.

GERSHUNY, Jonathan; BITTMAN, Michael; BRICE, John. "Exit, Voice, and Suffering: Do Couples adapt to Changing Employment Patterns?" In: ESRC RESEARCH CENTRE ON MICRO-SOCIAL CHANGE, 1997, Colchester: University of Essex. paper 97-98.

GERSHUNY, Jonathan; SULLIVAN, Oriel. "Time Use, Gender, and Public Policy Regimes." Social Politics, v. 10, n. 2, p. 205-228, 2003.

IBGE. Teste da pesquisa de uso do tempo: pesquisa nacional por amostra de domicílios/ PNAD contínua (versão em discussão). Rio de Janeiro: IBGE, 2009.

MICHELSON, William. Time Use: Expanding the Explanatory Power of the Social Sciences. London: Paradigm Publishers, 2005.

MILOSAVLEJIC, Vivian; TACLA, Odette. "Las encuestas del uso del tiempo: su diseño y aplicación". In: ORGANIZACIÓN PANAMERICANA DE LA SALUD. La economia invisible y las desigualdades de género: la importancia de medir y valorar el trabajo no remunerado. Washington, D.C.: OPS, 2008. p. 151-164.

OKIN, Susan. "Gender, the Public and the Private." In: PHILLIPS, Anne (Ed.). Feminism \& Politics. Oxford: Oxford University Press, 1998. p. 116-141.

PINHEIRO, Luana et al. Retrato das desigualdades de gênero e raça. 3. ed. Brasília: Ipea; SPM: UNIFEM, 2008.

PISCITELLI, Adriana. "Ambivalência sobre os conceitos de sexo e gênero na produção de algumas teóricas feministas". In: AGUIAR, Neuma (Org.). Gênero e ciências humanas: desafio às ciências desde a perspectiva das mulheres. Rio de Janeiro: Record; Rosa dos Tempos, 1997. p. 49-66. 
SALVADOR, Soledad. "La valoración económica del trabajo no remunerado". In: AGUIRRE, Rosario (Ed.). Las bases invisibles del bienestar social. Montevideo: Doble clic editoras, 2009. p. 155-204.

SORJ, Bila. "Percepções sobre esferas separadas de gênero". In: ARAÚJO, Clara; SCALON, Celi (Orgs.). Gênero, família e trabalho no Brasil. Rio de Janeiro: FGV, 2005. p. 79-88. SZALAl, Alexander (Ed.). The Use of Time. The Hague: Mouton, 1972.

\section{Time Use Research: A Tool to Measure Gender Inequalities}

Abstract: This paper analyses the interconnection between gender inequalities and time use, putting this analysis under the theoretic perspective of the time use surveys development and observing how it is reflected on the indicators of the Race and Gender Inequalities Picture. At first, time use surveys are characterized, and some of their history and current stage are recovered. The theoretic perspective that focuses on gender as a central category for the analysis of time use is also discussed. Some of the main results found by international time use surveys are presented, with an emphasis on the time use indicators elaborated on the basis of the Brazilian National Household Survey. Finally, it is suggested that time use surveys may contribute to foster public policies designed to attack gender inequalities.

Key Words: Time Use; Gender; Inequality; Unpaid Work. 